\title{
Face Detection in Video Using Local Spatio-temporal Representations
}

\author{
Yoanna Martínez-Díaz, Noslen Hernández, and Heydi Méndez-Vázquez \\ Advanced Technologies Application Center. 7th A Avenue $\sharp 21406 \% 214$ and 216, \\ Siboney, Playa, P.C. 12200, Havana, Cuba \\ \{ymartinez, nhernandez, hmendez\}@cenatav.co.cu
}

\begin{abstract}
Face detection is an important step in many video applications. Several algorithms have been proposed to this task, but most of them do not consider the spatio-temporal information. In this paper two recently introduced spatio-temporal descriptors are analyzed and evaluated in the context of face detection in videos. We designed and tested two full face detectors on the challenging YouTube Faces database. The obtained results are compared with those obtained by a frame-by-frame approach with a spatial descriptor, showing that using spatio-temporal descriptors can boost the detection performance.
\end{abstract}

Keywords: face detection, spatio-temporal representation, video.

\section{Introduction}

Face detection in video has become an important field of research with many applications such as face tracking, face recognition, facial expression analysis, human-computer interaction and video surveillance [12. It must be considered one of the key steps for any automatic video system; however, most of these tasks assume that the face has already been detected.

Among the different approaches described in the literature to solve the face detection problem in video, those that tackle the problem frame-by-frame have been the most used 410 . Nevertheless, these methods have been developed for still images not considering the explicit relationship that exists between consecutive frames. In this case each frame is analyzed as a new image without integrating any previous information, which can be also time consuming. In environments with static camera, motion detection algorithms have been applied in order to detect a face, however this kind of methods present poor performance in natural scenes [8].

Few attention has been paid to the integration of the temporal and spatial coherence of face appearance available in real sequences, although it has been shown the advantages of using local spatio-temporal descriptors in other topics of facial analysis [1]. For instance, the Volume Local Binary Patterns (VLBP) [13] proposed for facial expression recognition, the Extended set of VLBP (EVLBP) [5] used for face recognition and gender classification, and the Volume Structured Ordinal Feature (VSOF) 7] also introduced for facial recognition.

E. Bayro-Corrochano and E. Hancock (Eds.): CIARP 2014, LNCS 8827, pp. 860867 2014.

(C) Springer International Publishing Switzerland 2014 
Recently, a new approach was proposed for face/non-face classification in video sequences 6]. In such work, the authors shown that using the EVLBP spatio-temporal descriptor boosts the classification accuracy of AdaBoost algorithm, outperforming three approaches that use only the spatial information. Nevertheless, they only focused on deciding if a sequence is a face or a nonface, while in a realistic scenario the whole video should be analyzed using some scanning technique and each possible candidate region should be classified as face/non-face; returning the position of all faces in the video.

In this paper we propose spatio-temporal face detectors for video sequences. First, based on the the preliminary results obtained in [6], we develop a full EVLBP-based face detector. Second, motivated by the performance achieved by VSOF descriptor over EVLBP in video face recognition [7], we extend its application to the task of face detection in videos, designing a new VSOF-based face detector. Finally, we analyze and compare the performance of both detectors.

The remainder of this paper is organized as follows. Section 2 describes the local spatio-temporal EVLBP and VSOF descriptors used to represent the video sequences. In Section 3 we present the proposed spatio-temporal face detectors. Experimental results are provided in Section 4. Conclusions and future work are given in Section 5.

\section{Local Spatio-temporal Face Representations}

Most of existing local spatio-temporal descriptors proposed for facial analysis are extensions of popular local appearance based features to the video domain, by considering not only the spatial but also the temporal information. One example of this is the first extension of LBP descriptor to spatio-temporal domain, called Volume LBP (VLBP) [13. It consists of looking at a video sequence as a rectangular prism, and comparing each pixel not only with its neighboring pixels in the spatial domain, but also with those on its nearest frames. Afterwards, an Extended set of VLBP (EVLBP) features was introduced [5], being more flexible by using different radius, number of sampling points and sequence intervals.

The EVLBP operator is obtained for each pixel in every frame by [5]:

$$
E V L B_{L,(P, Q, S), R}=\sum_{m=0}^{M-1} s\left(I_{t, m}-I_{t_{c}, c}\right) 2^{m},
$$

where $t_{c}$ corresponds to the frame of the center pixel $c$ and $t$ is every frame used on the encoding process; $L$ is the time interval between encoded frames, and thus $t=t_{c}-2 L, t_{c}-L, t_{c}, t_{c}+L, t_{c}+2 L ; R$ is the radius for selecting the neighboring pixels; $M=P+2 Q+2 S$ is the total number of encoded pixels, chosen in the following way: $P$ pixels from frame $t, Q$ from frame $\pm t$ and $S$ from frame $\pm 2 t$; $I_{t_{c}, c}$ is the central pixel intensity value and $I_{t, m}$ is the intensity value of pixel $\mathrm{m}$ in frame $t ; s\{f\} \in\{0,1\}$ is a boolean indicator of the condition $f$.

Recently, the VSOF descriptor [7] inspired by the EVLBP one was proposed. It inherits the same flexible configuration but compares neighboring regions instead 
of pixels. In this way, more complex structures can be represented. The VSOF descriptor at a given scale $N$ (regions size $N \times N$ ) can be obtained by rewriting eq.(11), considering that $c$ is the central position of the center region and $g_{i}$ is the average intensity of region $i$. Thus, we have:

$$
V_{S O F},(P, Q, S), R, N=\sum_{m=0}^{M-1} s\left(g_{t, m}-g_{t_{c}, c}\right) 2^{m} .
$$

Notice that EVLBP is a special case of VSOF when $N=1$. For bigger scales, $N>1$, the "integral image" is used to compute the average intensity values from every block, reducing the computational cost. For both, EVLBP and VSOF descriptor, a large set of codes can be obtained by using different configurations of parameters. An example of one VSOF code obtained using $L=2, P=4$, $Q=3, S=1, R=3, N=3$, is illustrated in Figure 1.

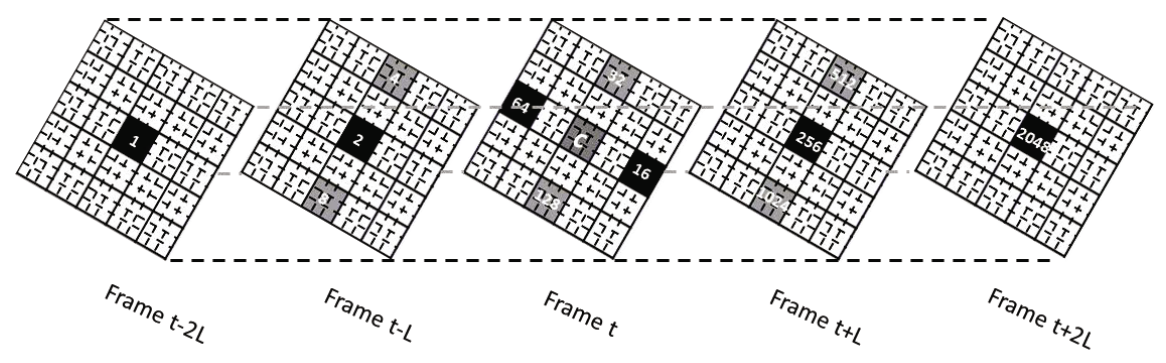

Fig. 1. Example of the VSOF operator encoding process. In this case $V S O F_{2,(4,3,1), 3,3}=110001011001=3161$.

In previous works [5/67 the distributions of EVLBP/VSOF codes (histograms) obtained with different configurations were used to describe video sequences. In this work, we also explore the use of the exhaustive set of codes as a feature vector to represent a face in a video.

\section{Spatio-temporal Face Detector}

It has been observed that the structure of any face presents a coherent spatial distribution, which it is conserved in time [2]. Thus, the permanence of certain features during a period of time could be exploited for detecting faces in video.

In this work we propose face detectors that take into account both the spatial and the temporal information available in video sequences. The structure of our detectors consist of: a spatio-temporal descriptor to represent face patterns, a boosting algorithm for automatically selecting and learning the most discriminative features and a cascade of boosting classifiers to speed up the detection process. 
We use EVLBP and VSOF descriptors to represent $M$ consecutive frames. The number of frames $M$ to be encoded places a key role in these representations. We need to ensure that only the face information is considered, but at the same time it is important to maximize the number of encoded frames to include more discriminative temporal information.

As mentioned in Section 2, both histograms and codes-based representations of EVLBP/VSOF can be used to describe faces in a video. In the first case, each feature from the complete vector corresponds to a bin of a histogram obtained for a given configuration, while in the second case represents the code with one configuration at a given position.

In both representations, several configurations of parameters can be used generating large feature sets. Hence, a boosting algorithm is applied in order to select the most discriminative features and reduce the redundant information. Many variants of boosting classifiers have been proposed in the literature for face detection [12. We adopt GentleBoost algorithm [3] because it is numerically robust and easy to implement. In the case of EVLBP/VSOF histograms, regression stumps can be used as weak classifier, while for EVLBP/VSOF codes, another kind of weak classifiers such as multibranch regression trees have to be adopted, because these codes are non-metric features and it is not possible to use threshold functions. The cascade of boosting classifiers is built following the Viola-Jones framework 9 .

For these spatio-temporal detectors, instead of a sliding window, we will have a sliding prism of depth $M$ for searching over an entire video at different scales and locations, determining whether a location corresponds to a face or not. Therefore, in this case we just need to scan a video every $M$ frames, which reduces significatively the computation time compared to the frame-by-frame algorithms. Once a cube is classified as a face, we will have the same detection for each of the $M$ frames that belongs to the detected cube. In the same way, if the detector fails we will lose the face during $M$ frames.

In addition, a post-processing method was proposed to merge detections that seem to be pointing to the same object. To achieve this we cluster the entire detections based on a dissimilarity measure that takes into account two key aspects: a) the area shared by two detections, not only in terms of quantity, but also in terms of significance of such area inside the detection windows being compared and $\mathrm{b}$ ) the difference in scale between detections. To ensure the first aspect we have a distribution of region's importance, $G_{s}$, for the detection template at each scale $s$, such that the closer the region to the template center, the most important it is (this was done using a bivariate Gaussian density). In this way, the location of the intersection area inside each detection is taken into account. The dissimilarity measure for two detections $d_{i}$ and $d_{j}$ can be formalize as,

$$
D\left(d_{i}, d_{j}\right)=1-\left(1 / 2\left(G_{s_{d_{i}}}\left(I\left(d_{i}, d_{j}\right)\right)+G_{s_{d_{j}}}\left(I\left(d_{i}, d_{j}\right)\right)\right) p\left(s_{d_{i}}, s_{d_{j}}\right)\right),
$$

where $s_{d_{i}}$ and $s_{d_{j}}$ are the scales of $d_{i}$ and $d_{j}$ respectively; $G_{s_{d_{i}}}, G_{s_{d_{j}}}$ give the contribution of the intersection area $I\left(d_{i}, d_{j}\right)$ and $p\left(s_{d_{i}}, s_{d_{j}}\right)$ penalizes the difference in scale. The greater the difference, the greater the penalization and 
consequently the lower the value of $p\left(s_{i}, s_{j}\right)$ ( $p$ ranges in the interval $\left.(0-1]\right)$. Note that the dissimilarity $D\left(d_{i}, d_{j}\right)$ takes value zero when $d_{i}$ and $d_{j}$ completely agree and one when $d_{i}$ and $d_{j}$ do not intersect. The clustering algorithm used was average-linkage. Once obtained the clusters, those with less than three detections are removed. Afterwards, the detection within each cluster with the highest confidence values is only considered. The confidence of the detection is the value of the strong classifier.

\section{Experimental Results and Discussion}

In case of face detection in videos it remains difficult to compare different algorithms, due to the lack of common evaluation protocols. Besides, most of the existing video databases were not specifically designed for this task and do not capture some aspects that are manifested in real-world scenarios. Hence, a public benchmark for face detection in video is not available.

For these reasons, we have decided in this work to conduct our experiments in the challenge YouTube Faces database [1], which contains 3425 videos of 1595 different subjects with different expressions, illuminations, poses, resolutions, and backgrounds.

In this section we first evaluate the discriminative power of the described EVLBP and VSOF descriptors. Then, the best performing type of features will be use to train the full detectors that are finally evaluated on a real scenario.

\subsection{Features Comparison}

This experiment compares histogram and codes-based representations for both EVLBP and VSOF descriptors. Hence, it is only restricted to decide whether a sample is a face or a non-face.

For this purpose, we built positive and negative rectangular prisms (or cubes) using $M=14$ frames, similar to [6]. Positive prims are selected using the face annotation given by the database in each of the $\mathrm{M}$ frames. Since for a given video, the face is not in the same position, the real volume formed by these annotated faces does not necessary form a rectangular prism. Thus, in order to capture as much as possible the real displacements of faces, we extract the rectangular prism with the greatest intersection with the real volume. In the case of negatives, the rectangular prims were obtained randomly from background areas.

In total we collected 40000 positive and 70000 negative cubes of size $40 \times 40 \times 14$ pixels, from 1000 videos of the database. These sets were divided in two parts; one part composed by 10000 positives and 10000 negatives cubes was used for training and the rest was used for testing. The feature extraction of the EVLBP and VSOF descriptors follows the explanation in Section 2 using many different parameter configurations. The length of the obtained feature vectors are given in Table 1, as well as the average time consumed to extract each of them. The GentleBoost algorithm consisting of 100 weak learners is used.

The performance of the different spatio-temporal representations evaluated is shown in Table 1, in terms of the False Negatives Rate (FN) and the False 
Table 1. Comparison of different types of spatio-temporal descriptors by using GentleBoost algorithm

\begin{tabular}{lcccc}
\hline Feature type & Vector length's & Extraction time $(\mathrm{sec})$ & $\mathrm{FN}(\%)$ & $\mathrm{FP}(\%)$ \\
\hline EVLBP-code & 78720 & 0.012 & 1.45 & 1.60 \\
EVLBP-histogram & 2048 & 0.055 & 3.10 & 6.14 \\
SOVLBP-code & 157056 & 0.031 & 1.15 & 1.46 \\
SOVLBP-histogram & 6144 & 0.121 & 1.92 & 4.99 \\
\hline
\end{tabular}

Positives Rate (FP). In general, the VSOF descriptor obtains better results than EVLBP descriptor. As it can be appreciated, for both EVLBP and SOVLBP, using directly the codes outperforms the histogram representation. On the other hand, working with EVLBP/VSOF histograms is more time consuming than with the codes, since they involve a further step. Besides, in the classification step, where only the selected features need to be computed, they require previously obtain all codes for the corresponding histogram configuration.

Considering the obtained results, we select the codes-based representation for both descriptors to train our face detectors.

\subsection{EVLBP and VSOF Detectors Evaluation}

In this experiment we trained two face detectors using the cascade of classifiers technique: one for EVLBP codes representation and the other for VSOF codes representation. The procedure is the same for both detectors, they only differ in the features.

For training we choose 21350 face cubes from the previous experiment, and 21350 non-face cubes, which are collected from $\approx 3000$ videos downloaded from Internet with no faces. After each stage is trained, the bootstrap strategy [9] is used to get the misclassified non-face cubes generated by the partial trained cascade that will be used to train the next stage. The maximum false alarm rate and the minimum detection rate were set as 0.5 and 0.995 respectively.

Our trained detectors have both 14 layers. The EVLBP detector includes 114 features, while the VSOF one contains 98 . We evaluate the performance of the detectors and compare them with a LBP detector applied frame-by-frame. For this purpose, we use OpenCV implementation and we get a LBP detector of also 14 layers with 82 features.

The evaluation dataset used consists of 95 videos from the database different to those used for training. The selected videos have only one face (the labeled one), since those videos that contain not labeled faces are not suitable for the face detection evaluation task. Each video has different amount of frames and thus we have in total, $\approx 23000$ images, all of them with only one individual but in challenging conditions. All videos were scanned using sliding prism of depth 14 and a scale factor of 1.25 for the pyramid detection.

In order to represent the degree of match between a detection $d_{i}$ and a groundtruth annotation $g_{i}$, we employ the commonly used ratio of intersected areas to 
Table 2. Performance of different detectors on the YouTube Faces database

\begin{tabular}{lcc}
\hline & Correct Detection (\%) & False Alarms \\
\hline EVLBP-detector & 86.67 & 8589 \\
VSOF-detector & 91.23 & 8332 \\
LBP-detector & 84.55 & 46643 \\
\hline
\end{tabular}
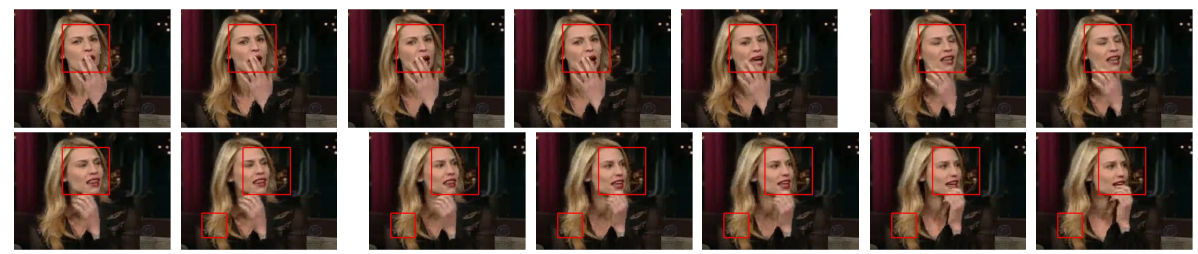

(a)
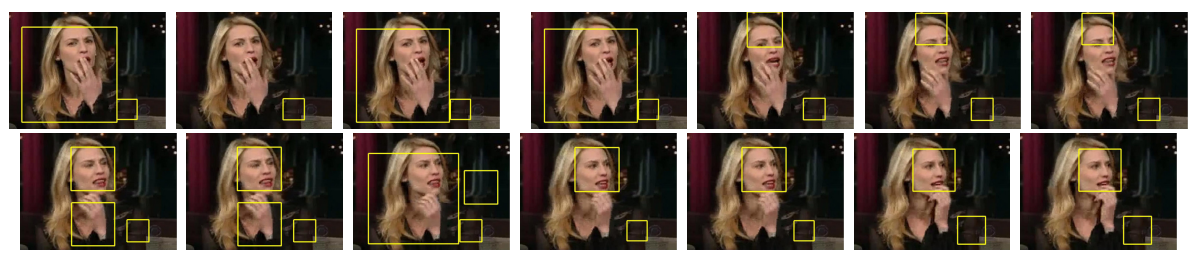

(b)

Fig. 2. Detections obtained using the VSOF detector (a) and the LBP detector (b) in a video sequence

joined areas. The resulting similarity score is compared to a given threshold $(0.5$ in this case) to decide whether a face is considered correctly detected or not.

Table 2 presents the results obtained for our two detectors and the LBP detector. It can be seen that both EVLBP and VSOF detectors outperform the LBP one, in terms of both correct detections and false alarms. Moreover it is shown that also in the case of face detection VSOF descriptor is more discriminative than EVLBP descriptor. In Figure 2 is illustrated the behavior of VSOF detector and the LBP one in 14 frames encoded in a cube sample. As we can appreciate, by using the proposed approach, a more robust position of the face in the sequence is obtained with less false positives.

\section{Conclusions and Future Work}

In this work we have proposed two face detectors for video sequences, which are based on the saptio-temporal information. We have shown that the used EVLBP and VSOF features are discriminative and robust for the face detection task under challenging conditions. Evaluations on the YouTube Faces database show that the proposed method outperforms frame-by-frame face detection algorithms. Although the detectors were only evaluated on videos that contained a single face, the system is able to detect multiple faces. 
Our future work will combine the spatio-temporal detector with some prediction technique which allow us to track the face on the complete video. Besides, we will explore the use of the same output of our spatio-temporal detector for face recognition in videos.

\section{References}

1. Barr, J.R., Bowyer, K.W., Flynn, P.J., Biswas, S.: Face Recognition from Video: a Review. IJPRAI 26(5) (2012)

2. Castrillón, M., Déniz, O., Guerra, C., Hernández, M.: Encara2: Real-time detection of multiple faces at different resolutions in video streams. J. Vis. Comun. Image Represent. 18(2), 130-140 (2007)

3. Friedman, J., Hastie, T., Tibshirani, R.: Additive Logistic Regression: a Statistical View of Boosting. Annals of Statistics 28, 2000 (1998)

4. Froba, B., Kublbeck, C.: Face tracking by means of continuous detection. In: IEEE Conf. Comput. Vision Pattern Recognition (CVPR) Workshops (2004)

5. Hadid, A., Pietikäinen, M.: Combining appearance and motion for face and gender recognition from videos. Pattern Recogn. 42(11), 2818-2827 (2009)

6. Martinez-Diaz, Y., Mendez-Vazquez, H., Hernandez, N., Garcia-Reyes, E.: Improving faces/non-faces discrimination in video sequences by using a local spatiotemporal representation. In: International Conference on Biometrics (ICB), pp. 1-5 (June 2013)

7. Mendez-Vazquez, H., Martinez-Diaz, Y., Chai, Z.: Volume structured ordinal features with background similarity measure for video face recognition. In: International Conference on Biometrics (ICB), pp. 1-6 (June 2013)

8. Nascimento, J.C., Marques, J.S.: Performance evaluation for object detection algorithms for video surveillance. IEEE Transaction on Multimedia (2006)

9. Viola, P., Jones, M.J.: Robust real-time face detection. International Journal of Computer Vision 57(2), 137-154 (2004)

10. Wael, L.: Co-occurrence of local binary patterns features for frontal face detection in surveillance applications. EURASIP Journal on Image and Video Processing 2011 (2011)

11. Wolf, L., Hassner, T., Maoz, I.: Face recognition in unconstrained videos with matched background similarity. In: IEEE Conf. Comput. Vision Pattern Recognition, CVPR (2011)

12. Zhang, C., Zhang, Z.: A survey of recent advances in face detection. Tech. Rep. MSR-TR-2010-66, Microsoft Research,, Redmond, Washington (June 2010)

13. Zhao, G., Pietikainen, M.: Dynamic texture recognition using local binary patterns with an application to facial expressions. IEEE Transactions on Pattern Analysis and Machine Intelligence 29(6), 915-928 (2007) 